\title{
Schlafbezogene Atmungsstörungen bei Patienten mit Herzinsuffizienz: Epiphänomen oder wechselseitige Krankheitsbeeinflussung
}

\author{
Sleep-Disordered Breathing in Patients with Chronic Heart Failure: \\ Epiphenomenon or Bidirectional Relationship
}

Autoren

Institut
J. Zeller, A. Hetzenecker, M. Arzt

Schlafmedizinisches Zentrum, Klinik und Poliklinik für Innere Medizin II, Universitätsklinikum Regensburg (Direktor der Klinik: Prof. Dr. med. G. A. J. Riegger) eingereicht 27.12 .2012

akzeptiert 15.1.2013

\section{Bibliografie}

DOI http://dx.doi.org/

10.1055/s-0032-1326221

Online-Publikation: 19.2.2013

Pneumologie 2013; 67: 150-156

(c) Georg Thieme Verlag KG

Stuttgart · New York

ISSN 0934-8387

\section{Korrespondenzadresse} Prof. Dr. med. Michael Arzt

Schlafmedizinisches Zentrum der Klinik und Poliklinik für Innere Medizin II

Universitätsklinikum Regensburg Franz-Josef-Strauß-Allee 11 93053 Regensburg michael.arzt@ukr.de

Serienherausgeber A. Valipour, Wien J. Ficker, Nürnberg

\section{Zusammenfassung \\ $\nabla$}

Schlafbezogene Atmungsstörungen (SBAS) stellen mit einer Prävalenz von ca. $45 \%$ eine sehr häufige Komorbidität der Herzinsuffizienz (HI) dar. Die beiden Erkrankungen sind wechselseitig voneinander abhängig: Einerseits kann die obstruktive Schlafapnoe zur Entstehung einer HI beitragen. Die Apnoe-assoziierte Erhöhung der kardialen Nachlast, die Entwicklung einer arteriellen Hypertonie und negative intrathorakale Drücke können zu einer Myokardhypertrophie und somit zu einer HI führen. Außerdem erhöht die Schlafapnoe das Herzinfarktrisiko und begünstigt die Entstehung einer HI nach Herzinfarkt. Anderseits kann die HI zur Entstehung sowohl der zentralen als auch der obstruktiven Schlafapnoe beitragen. Die optimale Behandlung einer bestehenden $\mathrm{HI}$ ist daher ein wichtiger Teil der Therapie einer SBAS. Die Therapie einer SBAS mittels positivem Atemwegsdruck reduziert effektiv Apnoen und Hypopnoen, verbessert den Schlaf und kann sich positiv auf das Befinden, die Lebensqualität und die Herzfunktion der Betroffenen auswirken. Die hohe Koinzidenz der SBAS und der HI rechtfertigt daher eine schlafmedizinische Diagnostik und Evaluation der Therapieindikation der SBAS in einem spezialisierten Zentrum.

\section{Einleitung \\ $\nabla$}

Schlafbezogene Atmungsstörungen (SBAS) und die chronische Herzinsuffizienz (HI) sind häufige Erkrankungen. Die Häufigkeit beider Erkrankungen ist stark altersabhängig. Vor allem die Prävalenz einer überwiegend zentralen Schlafapnoe (ZSA) steigt sowohl bei Männern als auch bei Frauen, ähnlich wie die Prävalenz der HI, ab einem Alter von ca. 65 bzw. 75 Jahren sprunghaft an.

\section{Abstract \\ $\nabla$}

Sleep-disordered breathing (SDB) constitutes a highly prevalent comorbidity in patients with chronic heart failure (HF, approximately $45 \%$ ). Both diseases are related in a bidirectional way: Obstructive sleep apnoea (OSA) can contribute to the development of HF via multiple mechanisms. Apnoea-related acute rise of cardiac afterload as well as manifest hypertension may contribute to the development of myocardial hypertrophy and thus HF. In addition, OSA increases the risk for myocardial infarction and impaired recovery of cardiac function after the event. Impaired cardiac function itself may contribute to the development of obstructive and central sleep apnoea (SA). Therefore, optimal medical management of HF is part of the therapy of SDB in such patients. Treatment of SDB with different modes of positive airway pressure suppresses apnoeas and hypopnoeas, improves sleep and may improve related symptoms and cardiac function of affected patients. Considering the high coincidence of SDB and HF, the adequate diagnosis of SDB and evaluation of indication for therapy of SDB performed in a specialised centre is advised.

Bei Patienten mit HI ist die Prävalenz von SBAS (Apnoe-Hypopnoe Index, AHI $\geq 15 / h$ ) mit ca. 45\%, auch unter Berücksichtigung der Altersabhängigkeit, mindestens doppelt so hoch wie bei Herzgesunden. Besteht bei Patienten mit HI entweder eine obstruktive, eine zentrale oder wie in dieser Patientengruppe häufig eine kombinierte Schlafapnoe, ist das Mortalitätsrisko um das Zwei- bis Dreifache erhöht. In Beobachtungsstudien haben Patienten mit HI und mit „Positive Airway Pressure“ (PAP) behandelter schwerer SBAS eine deutlich bessere Langzeitprognose als Patienten, die keine Behandlung der SBAS erhielten. 


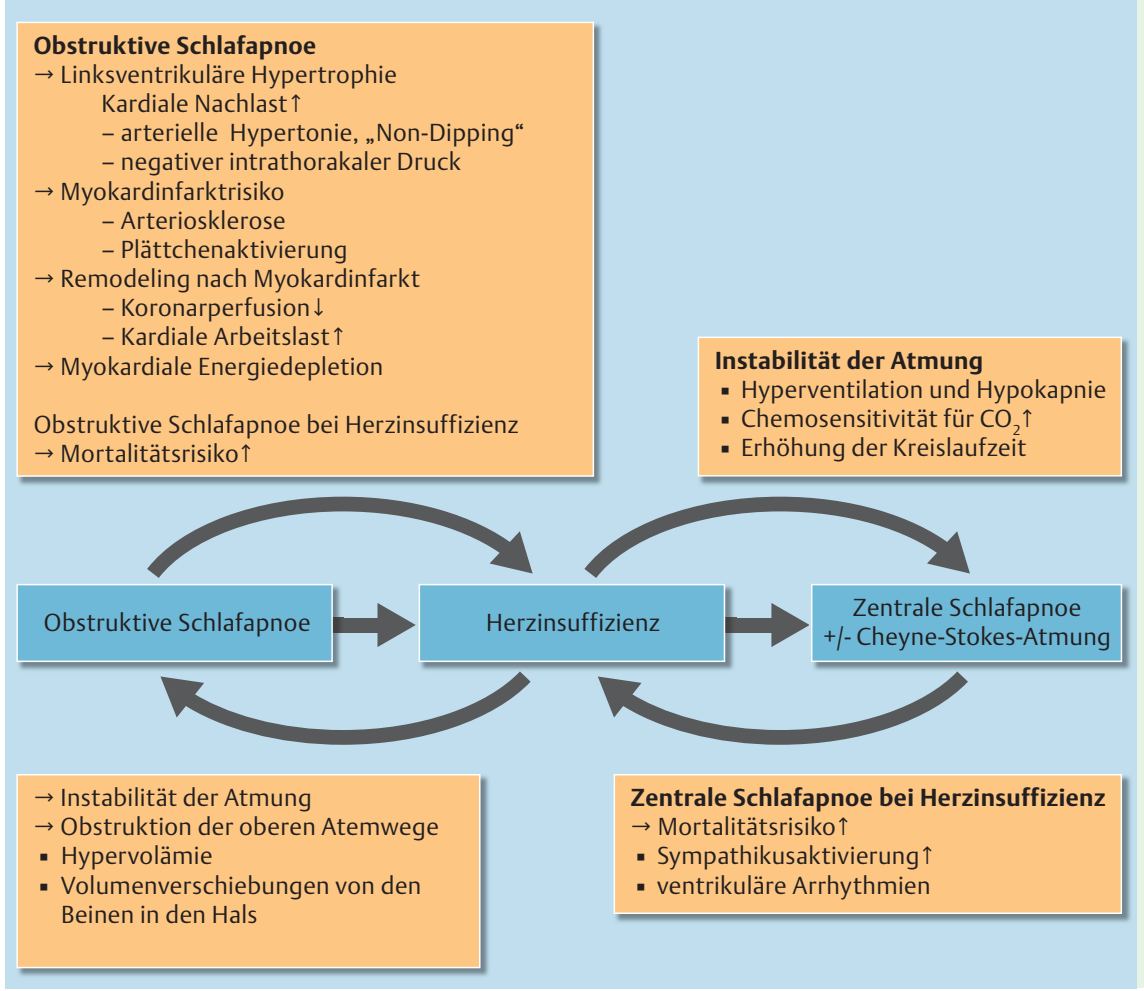

Abb. 1 Gegenseitige Wechselwirkung zwischen obstruktiver Schlafapnoe, Herzinsuffizienz und zentraler Schlafapnoe.
Diese Fakten legen eine gegenseitige kausale Krankheitsbeeinflussung und damit eine mögliche nicht-pharmakologische Therapie der HI durch Behandlung der SBAS nahe. Allerdings ist es in der bislang einzigen größeren, randomisierten Langzeitstudie auf diesem Gebiet nicht gelungen, durch eine Therapie der ZSA mit „Continuous Positive Airway Pressure“ (CPAP) bei Patienten mit HI die Hospitalisierungsrate und das Überleben zu verbessern. Dies lässt die Stimmen nicht verstummen, die vor allem die ZSA nur für ein Epiphänomen halten, dessen Behandlung die Progression der HI nicht beeinflusst. Vor diesem Hintergrund werden im Folgenden die komplexen Interaktionen von SBAS und der HI diskutiert ( $\bullet$ Abb. 1).

\section{Einfluss der obstruktiven Schlafapnoe auf die Entstehung und Progression der Herzinsuffizienz $\nabla$}

Linksventrikuläre Hypertrophie durch arterielle Hypertonie und Nachlasterhöhung

Die obstruktive Schlafapnoe (OSA) kann durch mehrere akute und chronische Effekte die Entstehung einer HI begünstigen oder deren Verlauf verschlechtern. Im Rahmen der repetitiven obstruktiven Apnoen kommt es zu intermittierender Hypoxie, Aufwachreaktionen und wiederholter Aktivierung des sympathischen Nervensystems mit Anstieg der Herzfrequenz und des arteriellen Blutdrucks. Zusätzlich zu den repetitiven Blutdruckanstiegen tragen die charakteristischen Atemanstrengungen gegen den verschlossenen Pharynx durch hohe negative intrathorakale Druckschwankungen, mit Spitzendrücken bis zu -65 mmHg, zu einem Anstieg des linksventrikulären transmuralen Drucks und damit zu einer Erhöhung der kardialen Nachlast bei $[1,2]$.

Prognostisch ist hinsichtlich der Entstehung einer Myokardhypertrophie [3] und der Mortalität [4] vor allem der fehlende nächtliche Blutdruckabfall („Non-Dipping“) entscheidend. Dieses
Phänomen des „Non-Dipping“ kann auch bei normotensiven Patienten mit OSA beobachtet werden und korreliert in seinem Ausmaß mit dem Schweregrad der Schlafapnoe [5]. Die OSA führt neben der akuten nächtlichen Blutdruckerhöhung auch zur Entstehung einer arteriellen Hypertonie, welche durch eine CPAPTherapie vor allem bei Patienten mit schwerer Schlafapnoe und Tagesmüdigkeit zusätzlich zur antihypertensiven Medikation erfolgreich behandelt werden kann [6-9]. Die OSA ist als Ursache der schwer behandelbaren arteriellen Hypertonie und als häufige Ursache einer sekundären arteriellen Hypertonie anerkannt [6]. Als Folge dieser Mechanismen kann die Schlafapnoe zur Entstehung einer linksventrikulären Hypertrophie beitragen [10]. Dabei ist im Rahmen der OSA schweregradabhängig vor allem das interventrikuläre Septum betroffen. In unkontrollierten Studien ist die Schlafapnoe-assoziierte septumbetonte Myokardhypertrophie durch eine 6-monatige CPAP-Therapie zumindest partiell reversibel [10].

\section{Erhöhtes Myokardinfarktrisiko durch endotheliale Dysfunktion, Arteriosklerose und Thrombozyten- aktivierung} Weitere Faktoren, die im Rahmen einer OSA auftreten und in Folge die Entwicklung einer HI begünstigen können, stellen die endotheliale Dysfunktion und die Arteriosklerose dar: Bei Patienten mit OSA sind die Spiegel von vasoaktiven Substanzen beeinträchtigt. Beispielsweise sind die Spiegel des zirkulierenden Stickstoffmonoxid erhöht und die Endothelinspiegel erniedrigt, sodass die Stickstoffmonoxid-vermittelte Vasodilatation gestört ist [11]. Die Stickstoffmonoxid-vermittelte Vasodilatation kann bei Patienten mit OSA durch eine CPAP-Therapie gesteigert werden.

Die Schlafapnoe-typische intermittierende Hypoxie stellt hier einen wichtigen Trigger für die Freisetzung von Entzündungsmediatoren und Sauerstoffradikalen dar [11 - 14]. Bei verschiedenen Entzündungsmediatoren wie dem C-reaktiven Protein und Interleukinen sowie bei erhöhtem oxidativem Stress konnte eine 
Assoziation mit arteriosklerotischen Veränderungen der Gefäßwand und der Thrombozytenaktivierung nachgewiesen werden $[15,16]$.

Unabhängig von anderen bekannten Risikofaktoren für Ateriosklerose führt die OSA zu endothelialer Apoptose und zu einer gestörten Gefäßwandreparatur. Um diesen Einfluss zu quantifizieren, konnte in mehreren Studien eine Zunahme der IntimaMedia-Dicke der A. carotis bei Patienten mit OSA unabhängig von weiteren kardialen Risikofaktoren nachgewiesen werden $[12,13,17]$. Dieses Frühzeichen der Arteriosklerose ist in einer kleinen randomisierten Studie bei Patienten mit OSA ebenfalls durch eine CPAP-Therapie zum Teil reversibel [13].

In prospektiven Beobachtungsstudien konnte mehrfach gezeigt werden, dass Patienten mit unbehandelter schwerer OSA im Vergleich zu Personen ohne SBAS oder mit einer leichtgradigen SBAS ein etwa dreifach höheres Risiko für kardiovaskuläre Ereignisse wie Herzinfarkt und Schlaganfall unabhängig von bekannten Risikofaktoren aufweisen [14]. Dabei tritt der Myokardinfarkt bei Patienten mit OSA gehäuft nachts auf, während bei Patienten ohne OSA sich die meisten Myokardinfarkte in den Morgenstunden nach dem Erwachen ereignen [18]. Bisher gibt es keine randomisierte Interventionsstudie von ausreichender Größe, die definitiv nachweisen kann, dass eine Therapie der OSA das Risiko für kardiovaskuläre Ereignisse reduziert [19]. Die jüngst publizierte randomisierte Studie von Barbé und Kollegen war nicht groß genug, um nachzuweisen, dass eine Behandlung der Schlafapnoe mit CPAP die Inzidenz der arteriellen Hypertonie und von kardiovaskulären Ereignissen reduzieren kann [19]. Die Post-hocAnalyse ergab jedoch eine Normalisierung des Risikos für das Neuauftreten einer arteriellen Hypertonie oder von kardiovaskulären Ereignissen in der Gruppe mit regelmäßiger Nutzung der CPAP-Therapie [19].

Die arterielle Hypertonie sowie die koronare Herzerkrankung stellen die beiden Hauptrisikofaktoren für die Entwicklung einer HI dar [20]. Hetzenecker, Buchner und Kollegen konnten zeigen, dass Patienten mit SBAS in der Frühphase nach einem akuten Myokardinfarkt im Vergleich zu Patienten ohne SBAS einen erhöhten Blutdruck, eine gesteigerte Herzfrequenz und somit eine erhöhte kardiale Arbeitslast aufwiesen [21]. Hierdurch kann das kardiale Remodelling nach Myokardinfarkt negativ beeinflusst werden [22]. Ein weiterer Mechanismus, durch den die OSA zur Entstehung einer HI beitragen kann, ist die OSA-assoziierte myokardiale Energiedepletion, die in einer Pilotstudie durch CPAP reversibel erscheint [23] ( $\bullet$ Abb.1).

In der Sleep Heart Health Study konnte zum ersten Mal in einer Kohortenstudie mit Langzeitbeobachtung nachgewiesen werden, dass schwere SBAS das Risiko für das Auftreten einer HI bei Männern um $58 \%$ steigern [24].

\section{Schlafbezogene Atmungsstörungen als Folge der Herzinsuffizienz}

Umgekehrt kann die HI zum Auftreten oder zu einer Verschlechterung einer sowohl obstruktiven als auch zentralen Schlafapnoe führen $(\bullet$ Abb. 1).

\section{Pathophysiologie der zentralen Schlafapnoe}

Mehrere Mechanismen begünstigen das Auftreten zentraler Atemereignisse im Rahmen einer chronischen HI. Erhöhte linksventrikuläre Füllungsdrücke können zu einer pulmonalvenösen Stauung mit Stimulation pulmonaler vagaler Rezeptoren führen.
Zusammen mit der für Patienten mit HI typischen erhöhten zentralen und peripheren Chemosensitivität für $\mathrm{CO}_{2}$ kommt es häufig zu einer chronischen Hyperventilation und Hypokapnie [2528]. Diese Hyperventilation mit Absinken des Kohlendioxidpartialdrucks unter die Apnoeschwelle, die oft durch die typischen Aufweckreaktionen (Arousals) provoziert wird, stellt einen Trigger für die zentrale Atempause dar [29]. In der Apnoephase steigt der Kohlendioxidpartialdruck wieder an und povoziert aufgrund der erhöhten Chemosensitivität für $\mathrm{CO}_{2}$ eine überschießende Atemantwort. Die Hyperventilation treibt den Kohlendioxidpartialdruck erneut unter die Apnoeschwelle. Hyperventilation und Apnoephasen können sich zyklisch abwechseln, was bei einer Crescendo-Decrescendo-artigen Änderung des Atemflusses als periodische Atmung oder Cheyne-Stokes-Atmungsmuster bezeichnet wird.

Ähnlich wie die OSA verursacht die ZSA eine intermittierende nächtliche Hypoxie mit Aktivierung des sympathischen Nervensystems und Anstieg des Blutdrucks [30]. Im Gegensatz zur OSA verursacht die ZSA jedoch keinen negativen intrathorakalen Druck. Folglich ist der Einfluss der ZSA auf die linksventrikuläre Vor- und Nachlast geringer als bei der OSA.

\section{Wie kann die Herzinsuffizienz zur obstruktiven Schlafapnoe beitragen?}

In epidemiologischen Studien fällt auf, dass bei Herzinsuffizienzpatienten der Zusammenhang zwischen dem Schweregrad der OSA mit dem Körpergewicht weniger stark ausgeprägt ist als bei Herzgesunden [31] und andere Faktoren eine Rolle spielen müssen.

Tatsächlich kann eine HI selbst, wie andere Erkrankungen oder Zustände, die mit einer Hypervolämie einhergehen wie z.B. die Niereninsuffizienz oder die Schwangerschaft, zum Auftreten oder zur Verstärkung einer bestehenden OSA beitragen. Die obere Atemwegsobstruktion im Rahmen der HI kommt hierbei in erster Linie durch nächtliche Flüssigkeitsverlagerungen zustande [32]. Flüssigkeit, die sich tagsüber in den Beinen angesammelt hat, verlagert sich durch die liegende Position während des Schlafes in den Bereich des Halses und führt dort durch Erweiterung der Jugularvenen und Ödembildung im Bereich des zervikalen Bindegewebes und der peripharyngealen Schleimhäute zur Einengung der Atemwege. Diese Ergebnisse konnten vor allem bei schlanken, männlichen, nicht-adipösen Probanden [32] oder Patienten mit OSA gezeigt werden. Dabei ist die Zunahme des Halsumfangs als Ausdruck der Flüssigkeitsverlagerung mit einem erhöhten AHI assoziiert [32]. Diese Flüssigkeitsverlagerungen korrelierten direkt mit dem Ausmaß der Beinödeme und der Zeit, die im Sitzen verbracht wurde, sowie mit dem Ausmaß der physischen Inaktivität [33].

\section{Effekte einer Behandlung der Herzinsuffizienz auf schlafbezogene Atmungsstörungen}

Die Intensivierung der medikamentösen Therapie der HI führt zu einer signifikanten Reduktion oder sogar dem kompletten Sistieren von zentralen Atemereignissen [26]. Diese Effekte waren am stärksten ausgeprägt nach Gabe positiv inotroper Substanzen und Diuretika [34], aber auch nach erhöhter Gabe von ACE-Hemmern und Betablockern $[26,35]$.

Auch die Erhöhung des Herzminutenvolumens durch eine Schrittmachertherapie kann eine ZSA [36-38] oder OSA [39] bessern oder beseitigen. Beispiele sind die atriale Überstimula- 


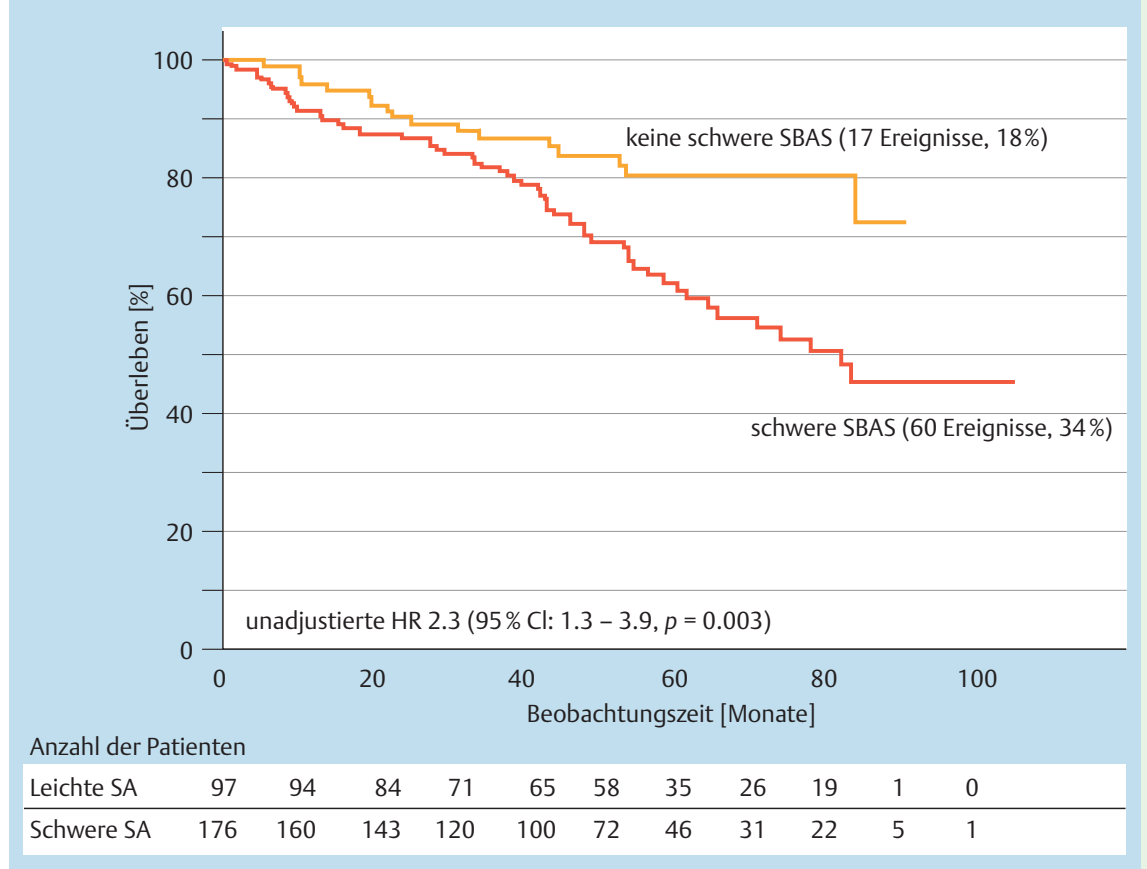

Abb.2 Kaplan-Meier-Plots für Patienten ohne schwere schlafbezogene Atemstörung (SBAS, Apnoe-Hypopnoe-Index $<22,5 / \mathrm{h}, \mathrm{n}=97$ ) und mit schwerer SBAS (Apnoe-Hypopnoe-Index $\geq 22,5 / \mathrm{h}$, $\mathrm{n}=176)$. Patienten mit chronischer $\mathrm{HI}$ ohne schwere SBAS hatten eine signifikant niedrigere Mortalität als Patienten mit schwerer SBAS. Unadjustiertes Cox-Proportional-Hazard-Modell. Abkürzungen: HR: Hazard Ratio; Cl: Konfidenzintervall [51].

tion bei Patienten mit Bradyarrhythmien [39] oder die kardiale Resynchronisationstherapie bei Patienten mit Kardiomyopathie [36-38].

Zusätzlich konnte gezeigt werden, dass sich nach Herzinfarkt durch Verbesserung der kardialen Funktion der Schweregrad der Schlafapnoe signifikant reduzieren ließ, wobei hier insbesondere die obstruktiven Atempausen vermindert werden konnten [40]. Eine weitere Studie erbrachte nach Normalisierung der Herzfunktion durch eine Herztransplantation eine signifikante Abnahme des Schweregrades der ZSA, wobei zwei Drittel der Patienten nach sechs Monaten keine zentralen Atemereignisse bzw. eine deutliche Besserung aufwiesen und ein Drittel der Patienten eine wahrscheinlich vorbestehende und durch die ZSA maskierte OSA zeigten $[41,42]$.

Diese Daten sprechen dafür, dass die Verbesserung der kardialen Funktion signifikant zur Stabilisierung der Atemkontrolle und Verbesserung der ZSA beiträgt. In einigen Studien konnte auch eine Reduktion der obstruktiven Atemereignisse gezeigt werden. Trotz der Implementierung wichtiger Therapien (Betarezeptorenblocker, ACE-Hemmer, Spironolacton oder Therapie mit biventrikulären Schrittmachern) blieb die Prävalenz schlafbezogener Atmungsstörungen insbesondere der ZSA in den letzten Jahren konstant [43]. Es zeigt sich jedoch, dass die unbehandelte HI zur Progression der ZSA beiträgt und somit die Therapieoptimierung der HI ein wichtiges Therapieprinzip bei Patienten mit ZSA darstellt.

\section{Prognostische Bedeutung schlafbezogener Atmungs- störungen bei Patienten mit Herzinsuffizienz

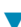

In mehreren Studien wurde die prognostische Bedeutung einer Schlafapnoe bei Patienten mit HI untersucht. Wang und Kollegen konnten in einer prospektiven Studie mit HI-Patienten nachweisen, dass unabhängig von bekannten Risikofaktoren die unbehandelte OSA (AHI über 15/h) mit einer höheren Mortalität einhergeht [44].
In mehreren Beobachtungsstudien konnte nachgewiesen werden, dass Patienten mit HI und unbehandelter schwerer ZSA unabhängig von bekannten Risikofaktoren eine erhöhte Mortalität im Vergleich zu Patienten ohne ZSA [45-47] und auch im Vergleich zu Patienten mit HI und OSA aufweisen [48-50]. Eine Ursache ist das erhöhte Risiko für das Auftreten von malignen ventrikulären Arrhythmien [50].

Jilek und Krenn mit Kollegen [51] konnten zeigen, dass Patienten mit chronischer HI und schwerer SBAS im Vergleich zu Patienten ohne schwere SBAS ein signifikant erhöhtes Mortalitätsrisiko aufweisen ( Abb.2). Gleichzeitig führt eine effektive PAP-Therapie mit CPAP- oder Adaptiver Servoventilation (in Ausnahmefällen mit Bilevel-PAP) zu einer signifikanten Reduktion des Mortalitätsrisikos im Vergleich zu unbehandelten Patienten ( $\bullet$ Abb. 3). Diese Studien hatten die wichtigen klinischen Prognosefaktoren bei HI wie die NYHA-Klassifikation, die linksventrikuläre Ejektionsfraktion, die medikamentöse Therapie sowie in einer Studie die mittels Ergospirometrie gemessene maximale Sauerstoffaufnahme [47] mitberücksichtigt, wobei sich bei einer schweren SBAS unabhängig von diesen Prognosefaktoren eine erhöhte Sterblichkeit nachweisen ließ. Die Ergebnisse dieser Studien legen einen kausalen Zusammenhang mit wechselseitiger Krankheitsbeeinflussung zwischen HI und Schlafapnoe nahe. Hinsichtlich der Auswirkungen einer PAP-Therapie auf die Prognose sind die Ergebnisse jedoch aufgrund der fehlenden Randomisierung nicht definitiv.

\section{Einfluss der Therapie schlafbezogener Atmungs- störungen auf die kardiale Funktion und die Prognose $\nabla$}

\section{Obstruktive Schlafapnoe}

Therapiestandard bei obstruktiver Schlafapnoe ist die Therapie mittels kontinuierlichem positiven Atemwegsdruck (CPAP). Dabei hat die CPAP-Therapie bei Patienten mit HI positive Effekte sowohl auf die pulmonale als auch auf die kardiale Funktion. 


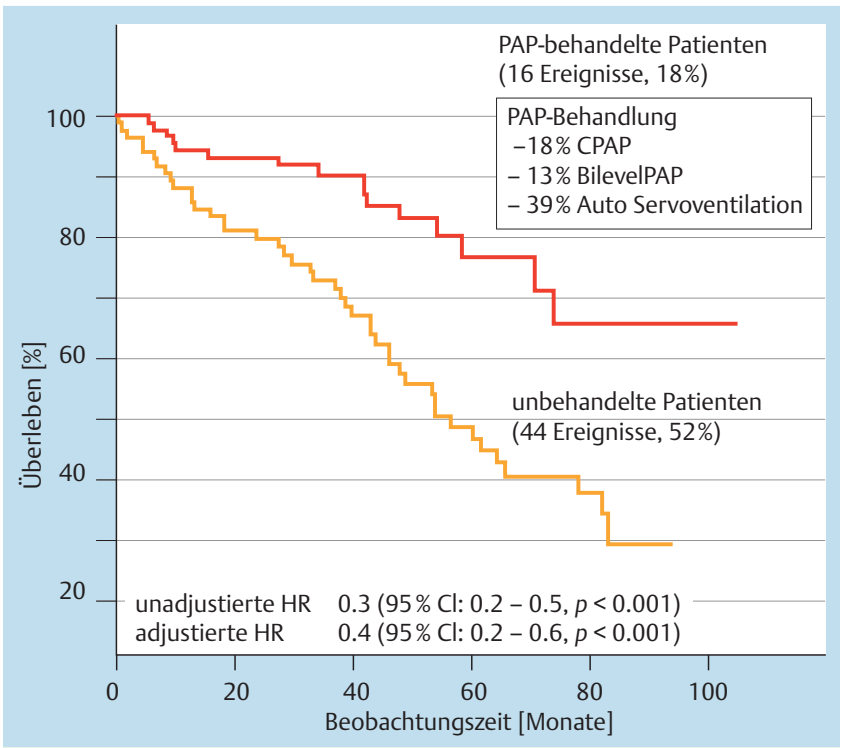

Abb. 3 Kaplan-Meier-Plots für Patienten mit schwerer schlafbezogener Atemstörung (SBAS, Apnoe-Hypopnoe-Index $\geq 22,5 / h)$ mit $(n=91)$ und ohne $(n=85)$ „Positive Airway Pressure“ (PAP)-Therapie. Sowohl im unadjustierten als auch im adjustierten Cox-Proportional-Hazard-Modell zeigten Patienten mit PAP-Therapie ein signifikant höheres Überleben als Patienten ohne PAP-Therapie. Das adjustierte Modell berücksichtigt auch die signifikanten Störvariablen Alter, NYHA-Klasse, Ursache der Herzinsuffizienz und Diabetes mellitus. Abkürzungen: HR: Hazard Ratio; Cl: Konfidenzintervall [51].

Positive Effekte auf die respiratorische Funktion

Im Rahmen der HI treten gehäuft obstruktive Ventilationsstörungen durch ein alveoläres Lungenödem sowie restriktive Ventilationsstörungen durch Vergrößerung des Herzens, Pleuraergüsse oder eine stauungsbedingte Volumenzunahme des Abdomens auf [52-54]. Diese Ventilationsstörungen können durch die CPAP-Therapie, neben den Effekten auf die SBAS, positiv beeinflusst werden. Die CPAP-Therapie erhöht den alveolären Druck und verhindert somit einen Alveolarkollaps, der durch ein alveoläres Lungenödem hervorgerufen werden kann. Gleichzeitig wird der Gasaustausch verbessert, das Lungenvolumen erhöht [55] und Ventilations-/Perfusionsstörungen beseitigt [56]. Zudem erhöht die CPAP-Therapie den Durchmesser der Atemwege durch eine Reduktion des Bronchialwandödems [57]. Somit sinkt die respiratorische Arbeit sowohl während des Wachzustandes [57] als auch während des Schlafs.

\section{Kardiovaskuläre Effekte}

Die CPAP-Therapie führt zu einer mechanischen Schienung der oberen Atemwege und reduziert dadurch die negativen Auswirkungen der OSA, wie die intermittierende nächtliche Hypoxie und Hyperkapnie, den erhöhten arteriellen Blutdruck sowie den erhöhten Sympathikotonus [1,58]. Dabei verbessert die chronische Anwendung der CPAP-Therapie langfristig die kardiovaskuläre Funktion. Durch Aufbau eines positiven intrathorakalen Drucks verringert die CPAP-Therapie die kardiale Vorlast. Da Patienten mit HI häufig volumenüberladen sind und hohe Füllungsdrücke aufweisen, wirkt sich hier die Reduktion des venösen Rückstroms zum Herzen besonders positiv aus. Dies kommt durch eine deutliche Zunahme des Schlagvolumens zum Ausdruck.

Zusätzlich senkt die CPAP-Therapie die kardiale Nachlast durch Vermeidung der mit den obstruktiven Apnoen verbundenen Druckschwankungen, wodurch ein positiver Druck um den lin- ken Ventrikel gebildet wird, der zu einer Abnahme der Wandspannung führt [57].

Malone und Kollegen konnten bei Patienten mit HI und OSA nach einmonatiger CPAP-Therapie eine Verbesserung der linksventrikulären Ejektionsfunktion von 37 auf 49\% mit Verbesserung der NYHA-Klasse erreichen [2]. Eine Verbesserung der Ejektionsfraktion um 9\% und eine signifikante Reduktion des arteriellen Blutdrucks nach ebenfalls einmonatiger CPAP-Therapie konnten Kaneko und Kollegen in einer randomisierten Studie im Vergleich zu einer Kontrollgruppe mit optimaler medikamentöser Herzinsuffizienztherapie nachweisen [59].

Neben der Verbesserung der linksventrikulären Funktion führt die CPAP-Behandlung zu einer Reduktion der sympathischen Muskelaktivität [53], der ventrikulären Ektopierate im Schlaf [60] und zu einer erhöhten kardialen parasympathischen Aktivität $[61,62]$.

\section{Zentrale Schlafapnoe}

In mehreren kleineren Studien konnte gezeigt werden, dass bei ZSA unter CPAP-Therapie mit Drücken von $8-12,5 \mathrm{~cm} \mathrm{H}_{2} 0$ die linksventrikuläre Pumpfunktion [63-65], die Regurgitationsfraktion über die Mitralklappe [66], die Sympathikusaktivität, gemessen an erniedrigten Norepinephrin-Spiegeln [55], und das Ausmaß der HI, gemessen an erniedrigten ANP-Spiegeln [66], verbessert werden konnten. Inwieweit die CPAP-Therapie die kardiale Funktion bei Patienten mit ZSA verbessert, wurde in der CANPAP-Studie, einer randomisierten Langzeit-Multizenterstudie untersucht [67]. Hier konnte eine moderate Steigerung der linksventrikulären Pumpfunktion, eine Verbesserung der nächtlichen Oxygenierung und eine Reduktion der Norepinephrinspiegel nachgewiesen werden. Insgesamt konnten keine signifikanten Unterschiede im transplantatfreien Überleben, der Hospitalisierungsrate und der Lebensqualität der Patienten festgestellt werden. In einer Post-hoc-Analyse zeigte sich, dass die Patienten mit einer effektiven CPAP-Therapie mit Erreichen eines AHI unter 15 Ereignissen pro Stunde eine signifikant stärkere Verbesserung der linksventrikulären Funktion und des transplantatfreien Überlebens aufwiesen, verglichen mit den Patienten mit einem AHI über 15 Ereignissen pro Stunde und der Kontrollgruppe [68]. Speziell für das Atemmuster der Cheyne-Stokes-Atmung im Rahmen einer ZSA wurde die adaptive Servoventilation (ASV) entwickelt. Hierbei passt sich die Druckunterstützung der variierenden Spontanatmung des Patienten an. Es gibt derzeit noch keine randomisierten Überlebens- oder Langzeitdaten für die ASV-Therapie bei Patienten mit HI. Dennoch scheint die ASV-Therapie in dieser Patientengruppe der CPAP-Therapie überlegen, da sie zu einer effektiveren Reduktion der zentralen Atemereignisse führt. In Metaanalysen konnte nachgewiesen werden, dass die ASV den AHI um 12-23/h stärker verbessert als die CPAP-Therapie [69, 70]. Die bisherigen kleinen randomisierten Studien zeigen widersprüchliche Ergebnisse hinsichtlich der Effekte der ASV auf die linksventrikuläre Ejektionsfraktion [70-74], jedoch senkt die ASV konsistent die NTproBNP- oder BNP-Werte als Ausdruck einer Reduktion der kardialen Füllungsdrücke und einer Besserung der Herzfunktion [71-73].

\section{Schlussfolgerung}

Insgesamt zeichnet sich ab, dass zwischen SBAS und der HI eine gegenseitige Krankheitsbeeinflussung vorliegt. Sowohl die OSA als auch die ZSA können zum Fortschreiten der HI beitragen und 
können durch eine CPAP-Therapie bzw. eine Therapie mit ASV, die bei der ZSA effektiver ist, erfolgreich behandelt werden. Eine effektive PAP-Therapie führt bei Patienten mit HI und symptomatischer SBAS in der Regel zu einer Besserung der Symptome. In Beobachtungsstudien haben Patienten mit behandelter schwerer SBAS eine bessere Prognose als Patienten mit einer unbehandelten SBAS. Es gibt jedoch bislang keine randomisierten Langzeitstudien, die einen positiven Einfluss auf die kardiovaskuläre Morbidität und Mortalität durch eine suffiziente Therapie der Schlafapnoe nachweisen. In größeren Studienpopulationen wie z. B. der SERVE-HF- und ADVENT-HF-Studie wird aktuell mit der effektiveren ASV-Therapie untersucht, ob eine Verbesserung der Herzfunktion und letztlich eine Prognoseverbesserung durch die Therapie der ZSA und OSA bei Patienten mit $\mathrm{HI}$ ohne relevante Schlafapnoe-assoziierte Symptome möglich ist. Die optimale Therapie der HI ist ein wichtiges Therapieprinzip bei Patienten mit HI und SBAS.

\section{Interessenkonflikt}

\section{$\nabla$}

J. Zeller und A. Hetzenecker geben an, dass kein Interessenkonflikt besteht. M. Arzt erhielt Forschungsunterstützung und Vortragshonorare von ResMed und Philips Respironics.

\section{Literatur}

1 Tkacova R, Rankin F, Fitzgerald FS et al. Effects of continuous positive airway pressure on obstructive sleep apnea and left ventricular afterload in patients with heart failure. Circulation 1998; 98: 2269-2275

2 Malone S, Liu PP, Holloway R et al. Obstructive sleep apnoea in patients with dilated cardiomyopathy: effects of continuous positive airway pressure. Lancet 1991; 338: 1480-1484

3 Verdecchia P, Schillaci G, Guerrieri $M$ et al. Circadian blood pressure changes and left ventricular hypertrophy in essential hypertension. Circulation 1990; 81: 528-536

4 Sega $R$, Facchetti $R$, Bombelli $M$ et al. Prognostic value of ambulatory and home blood pressures compared with office blood pressure in the general population: follow-up results from the Pressioni Arteriose Monitorate e Loro Associazioni (PAMELA) study. Circulation 2005; 111: $1777-1783$

5 Suzuki M, Guilleminault C, Otsuka K et al. Blood pressure „dipping“ and „non-dipping“ in obstructive sleep apnea syndrome patients. Sleep 1996; 19: $382-387$

6 Calhoun $D A$, Jones $D$, Textor $S$ et al. Resistant hypertension: diagnosis, evaluation, and treatment: a scientific statement from the American Heart Association Professional Education Committee of the Council for High Blood Pressure Research. Circulation 2008; 117: e510-526

7 Peppard PE, Young T, Palta M et al. Prospective Study of the Association between Sleep-Disordered Breathing and Hypertension. N Engl J Med 2000; 342: 1378 - 1384

8 Pepperell JCT, Ramdassingh-Dow S, Crosthwaite $N$ et al. Ambulatory blood pressure after therapeutic and subtherapeutic nasal continuous positive airway pressure for obstructive sleep apnoea: a randomised parallel trial. Lancet 2002; 359: 204-210

9 Becker HF, Jerrentrup A, Ploch T et al. Effect of nasal continuous positive airway pressure treatment on blood pressure in patients with obstructive sleep apnea. Circulation 2003; 107: 68-73

10 Shivalkar B, van de Heyning $C$, Kerremans $M$ et al. Obstructive sleep apnea syndrome: more insights on structural and functional cardiac alterations, and the effects of treatment with continuous positive airway pressure. J Am Coll Cardiol 2006; 47: 1433-1439

11 Ip MSM, Tse H, Lam B et al. Endothelial function in obstructive sleep apnea and response to treatment. Am J Respir Crit Care Med 2004; 169: $348-353$

12 Minoguchi K, Yokoe T, Tazaki $T$ et al. Increased carotid intima-media thickness and serum inflammatory markers in obstructive sleep apnea. Am J Respir Crit Care Med 2005; 172: 625-630

13 Drager LF, Bortolotto LA, Lorenzi MC et al. Early signs of atherosclerosis in obstructive sleep apnea. Am J Respir Crit Care Med 2005; 172: 613 618
14 Marin JM, Carrizo SJ, Vicente E et al. Long-term cardiovascular outcomes in men with obstructive sleep apnoea-hypopnoea with or without treatment with continuous positive airway pressure: an observational study. Lancet 2005; 365: 1046 - 1053

15 Ross R. Atherosclerosis - an inflammatory disease. N Engl J Med 1999; 340: $115-126$

16 Shimokawa $\mathrm{H}$. Primary endothelial dysfunction: atherosclerosis. J Mol Cell Cardiol 1999; 31: 23-37

17 Schulz R, Seeger W, Fegbeutel C et al. Changes in extracranial arteries in obstructive sleep apnoea. Eur Respir J 2005; 25: 69-74

18 Kuniyoshi FHS, Garcia-Touchard A, Gami AS et al. Day-night variation of acute myocardial infarction in obstructive sleep apnea. J Am Coll Cardiol 2008; 52: $343-346$

19 Barbé F, Durán-Cantolla J, Sánchez-de-la-Torre M et al. Effect of continuous positive airway pressure on the incidence of hypertension and cardiovascular events in nonsleepy patients with obstructive sleep apnea: a randomized controlled trial. JAMA 2012; 307: 2161 - 2168

20 McKee PA, Castelli WP, McNamara PM et al. The natural history of congestive heart failure: the Framingham study. N Engl J Med 1971; 285: $1441-1446$

21 Hetzenecker A, Buchner S, Greimel T et al. Cardiac workload in patients with sleep-disordered breathing early after acute myocardial infarction. Chest 2012: epub ahead of print

22 Nakashima H, Katayama T, Takagi C et al. Obstructive sleep apnoea inhibits the recovery of left ventricular function in patients with acute myocardial infarction. Eur Heart J 2006; 27: 2317-2322

23 Yoshinaga K, Burwash IG, Leech JA et al. The effects of continuous positive airway pressure on myocardial energetics in patients with heart failure and obstructive sleep apnea. J Am Coll Cardiol 2007; 49: 450 458

24 Gottlieb DJ, Yenokyan G, Newman AB et al. Prospective study of obstructive sleep apnea and incident coronary heart disease and heart failure: the sleep heart health study. Circulation 2010; 122: 352 - 360

25 Lorenzi-Filho G, Azevedo ER, Parker JD et al. Relationship of carbon dioxide tension in arterial blood to pulmonary wedge pressure in heart failure. Eur Respir J 2002; 19: 37-40

26 Solin P, Bergin P, Richardson $M$ et al. Influence of pulmonary capillary wedge pressure on central apnea in heart failure. Circulation 1999; 99: $1574-1579$

27 Arzt M, Harth M, Luchner A et al. Enhanced ventilatory response to exercise in patients with chronic heart failure and central sleep apnea. Circulation 2003; 107: 1998-2003

28 Tkacova R, Hall MJ, Liu PP et al. Left ventricular volume in patients with heart failure and Cheyne-Stokes respiration during sleep. Am J Respir Crit Care Med 1997; 156: 1549-1555

29 Naughton M, Benard D, Tam A et al. Role of hyperventilation in the pathogenesis of central sleep apneas in patients with congestive heart failure. Am Rev Respir Dis 1993; 148: 330-338

30 Leung RST, Floras JS, Lorenzi-Filho G et al. Influence of Cheyne-Stokes respiration on cardiovascular oscillations in heart failure. Am J Respir Crit Care Med 2003; 167: 1534-1539

31 Arzt M, Young T, Finn L et al. Sleepiness and sleep in patients with both systolic heart failure and obstructive sleep apnea. Arch Intern Med 2006; 166: 1716-1722

32 Redolfi S, Yumino D, Ruttanaumpawan $P$ et al. Relationship between overnight rostral fluid shift and Obstructive Sleep Apnea in nonobese men. Am J Respir Crit Care Med 2009; 179: 241 - 246

33 Yumino D, Redolfi S, Ruttanaumpawan $P$ et al. Nocturnal rostral fluid shift: a unifying concept for the pathogenesis of obstructive and central sleep apnea in men with heart failure. Circulation 2010; 121: $1598-1605$

34 Dark DS, Pingleton SK, Kerby GR et al. Breathing pattern abnormalities and arterial oxygen desaturation during sleep in the congestive heart failure syndrome. Improvement following medical therapy. Chest 1987; 91: 833-836

35 Walsh JT, Andrews R, Starling $R$ et al. Effects of captopril and oxygen on sleep apnoea in patients with mild to moderate congestive cardiac failure. Br Heart J 1995; 73: 237-241

36 Kato I, Shiomi T, Sasanabe R et al. Effects of physiological cardiac pacing on sleep-disordered breathing in patients with chronic bradydysrhythmias. Psychiatry Clin Neurosci 2001; 55: 257-258

37 Gabor JY, Newman DA, Barnard-Roberts $V$ et al. Improvement in Cheyne-Stokes respiration following cardiac resynchronisation therapy. Eur Respir J 2005; 26: 95-100 
38 Sinha A, Skobel EC, Breithardt $O$ et al. Cardiac resynchronization therapy improves central sleep apnea and Cheyne-Stokes respiration in patients with chronic heart failure. J Am Coll Cardiol 2004; 44: 68 - 71

39 Garrigue S, Bordier P, Jais $P$ et al. Benefit of atrial pacing in sleep apnea syndrome. N Engl J Med 2002; 346: 404-412

40 Buchner S, Greimel T, Hetzenecker A et al. Natural course of sleep-disordered breathing after acute myocardial infarction. Eur Respir J 2012; 40: $1173-1179$

41 Mansfield DR, Solin P, Roebuck T et al. The effect of successful heart transplant treatment of heart failure on central sleep apnea. Chest 2003; 124: 1675 - 1681

42 Javaheri S, Abraham WT, Brown C et al. Prevalence of obstructive sleep apnoea and periodic limb movement in 45 subjects with heart transplantation. Eur Heart J 2004; 25: 260-266

43 Yumino D, Wang H, Floras JS et al. Prevalence and physiological predictors of sleep apnea in patients with heart failure and systolic dysfunction. J Card Fail 2009; 15: 279-285

44 Wang H, Parker JD, Newton GE et al. Influence of Obstructive Sleep Apnea on Mortality in Patients With Heart Failure. Journal of the American College of Cardiology 2007; 49: 1625-1631

45 Javaheri S, Shukla R, Zeigler $H$ et al. Central sleep apnea, right ventricular dysfunction, and low diastolic blood pressure are predictors of mortality in systolic heart failure. J Am Coll Cardiol 2007; 49: 2028 2034

46 Lanfranchi PA, Braghiroli A, Bosimini E et al. Prognostic value of nocturnal Cheyne-Stokes respiration in chronic heart failure. Circulation 1999; 99: 1435 - 1440

47 Corra $U$, Pistono $M$, Mezzani A et al. Sleep and exertional periodic breathing in chronic heart failure: prognostic importance and interdependence. Circulation 2006; 113: 44-50

48 Bakker JP, Campbell AJ, Neill AM. Increased mortality risk in congestive heart failure patients with comorbid sleep apnoea: 10-year follow up. Intern Med J 2012; 42: 1264 - 1268

49 Damy T, Margarit L, Noroc A et al. Prognostic impact of sleep-disordered breathing and its treatment with nocturnal ventilation for chronic heart failure. Eur J Heart Fail 2012; 14: 1009-1019

50 Bitter T, Westerheide N, Prinz C et al. Cheyne-Stokes respiration and obstructive sleep apnoea are independent risk factors for malignant ventricular arrhythmias requiring appropriate cardioverter-defibrillator therapies in patients with congestive heart failure. Eur Heart J 2011; 32: $61-74$

51 Jilek C, Krenn M, Sebah D et al. Prognostic impact of sleep disordered breathing and its treatment in heart failure: an observational study. Eur J Heart Fail 2011; 13: 68-75

52 Light $R W$, George RB. Serial pulmonary function in patients with acute heart failure. Arch Intern Med 1983; 143: 429-433

53 Hosenpud JD, Stibolt TA, Atwal K et al. Abnormal pulmonary function specifically related to congestive heart failure: comparison of patients before and after cardiac transplantation. Am J Med 1990; 88: 493-496

54 Naum CC, Sciurba FC, Rogers RM. Pulmonary function abnormalities in chronic severe cardiomyopathy preceding cardiac transplantation. Am Rev Respir Dis 1992; 145: 1334-1338

55 Naughton MT, Benard DC, Liu PP et al. Effects of nasal CPAP on sympathetic activity in patients with heart failure and central sleep apnea. Am J Respir Crit Care Med 1995; 152: 473-479

56 Krachman SL, Crocetti J, Berger TJ et al. Effects of nasal continuous positive airway pressure on oxygen body stores in patients with CheyneStokes respiration and congestive heart failure. Chest 2003; 123: 5966
57 Naughton MT, Rahman MA, Hara K et al. Effect of continuous positive airway pressure on intrathoracic and left ventricular transmural pressures in patients with congestive heart failure. Circulation 1995; 91: $1725-1731$

58 Usui K, Bradley TD, Spaak J et al. Inhibition of awake sympathetic nerve activity of heart failure patients with obstructive sleep apnea by nocturnal continuous positive airway pressure. J Am Coll Cardiol 2005; 45: $2008-2011$

59 Kaneko Y, Floras JS, Usui $\mathrm{K}$ et al. Cardiovascular effects of continuous positive airway pressure in patients with heart failure and obstructive sleep apnea. N Engl J Med 2003; 348: 1233 -1241

60 Ryan CM, Usui $K$, Floras JS et al. Effect of continuous positive airway pressure on ventricular ectopy in heart failure patients with obstructive sleep apnoea. Thorax 2005; 60: 781 - 785

61 Ruttanaumpawan P, Gilman MP, Usui K et al. Sustained effect of continuous positive airway pressure on baroreflex sensitivity in congestive heart failure patients with obstructive sleep apnea. J Hypertens 2008; 26: $1163-1168$

62 Gilman MP, Floras JS, Usui K et al. Continuous positive airway pressure increases heart rate variability in heart failure patients with obstructive sleep apnoea. Clin Sci 2008; 114: 243-249

63 Sin DD, Logan AG, Fitzgerald FS et al. Effects of continuous positive airway pressure on cardiovascular outcomes in heart failure patients with and without Cheyne-Stokes respiration. Circulation 2000; 102: $61-66$

64 Arzt $M$, Schulz $M$, Wensel $R$ et al. Nocturnal continuous positive airway pressure improves ventilatory efficiency during exercise in patients with chronic heart failure. Chest 2005; 127: 794-802

65 Naughton MT, Liu PP, Bernard DC et al. Treatment of congestive heart failure and Cheyne-Stokes respiration during sleep by continuous positive airway pressure. Am J Respir Crit Care Med 1995; 151: 92 - 97

66 Tkacova R, Liu PP, Naughton MT et al. Effect of continuous positive airway pressure on mitral regurgitant fraction and atrial natriuretic peptide in patients with heart failure. J Am Coll Cardiol 1997; 30: 739745

67 Bradley TD, Logan AG, Kimoff RJ et al. Continuous positive airway pressure for central sleep apnea and heart failure. N Engl J Med 2005; 353: 2025-2033

68 Arzt M, Floras JS, Logan AG et al. Suppression of central sleep apnea by continuous positive airway pressure and transplant-free survival in heart failure: a post hoc analysis of the Canadian Continuous Positive Airway Pressure for Patients with Central Sleep Apnea and Heart Failure Trial (CANPAP). Circulation 2007; 115: 3173-3180

69 Aurora RN, Chowdhuri S, Ramar K et al. The treatment of central sleep apnea syndromes in adults: practice parameters with an evidencebased literature review and meta-analyses. Sleep 2012; 35: 17-40

70 Sharma BK, Bakker JP, McSharry DG et al. Adaptive servo-ventilation for treatment of sleep-disordered breathing in heart failure: A systematic review and meta-analysis. Chest 2012; 142: 1211-1221

71 Kasai T, Floras JS, Bradley TD. Sleep apnea and cardiovascular disease: a bidirectional relationship. Circulation 2012; 126: 1495-1510

72 Arzt M, Schroll S, Series F et al. Auto-servo ventilation in heart failure with sleep apnea - a randomized controlled trial. Eur Respir J 2012: epub ahead of print

73 Pepperell JC. Sleep apnoea syndromes and the cardiovascular system. Clin Med 2011; 11: 275-278

74 Randerath WJ, Nothofer G, Priegnitz C et al. Long-term auto-servoventilation or constant positive pressure in heart failure and coexisting central with obstructive sleep apnea. Chest 2012; 142: 440-447 